\title{
AUXÍLIO COMPUTADORIZADO À IDENTIFICAÇÃO DO CÂNCER DE PULMÃO BASEADO EM MAPAS DE CONEXIDADE FUZZY PARTINDO DA ESCOLHA DE UMA SEMENTE IDEAL
}

\author{
Tiago Emmanuel Praxedes Silva \\ Universidade Federal de Alagoas \\ Graduando em Ciência da Computação \\ teps19@gmail.com
}

\begin{abstract}
Marcelo Costa Oliveira
Professor do Instituto de Computação da Universidade Federal de Alagoas. Doutor em Ciências

Médicas na área de Informática Médica pela Universidade de São Paulo (USP)

oliveiramc@gmail.com
\end{abstract}

\section{RESUMO}

O objetivo deste trabalho foi avaliar de maneira qualitativa a precisão da segmentação por meio do algoritmo de construção de mapas de conexidade fuzzy partindo da escolha de uma semente ideal em relação à segmentação manual. Foram selecionados de maneira aleatória 30 nódulos do projeto "Lung Image Database Consortium". O algoritmo desenvolvido se mostrou eficaz em identificar os nódulos pulmonares atingindo $90,4 \%$ de precisão. Além disso, superou a precisão do tradicional algoritmo de segmentação por crescimento de região em $9,1 \%$. Logo, esta técnica possui potencial para ser utilizada como uma ferramenta de auxílio ao diagnóstico do câncer de pulmão.

PALAVRAS-CHAVE: segmentação de imagens, câncer de pulmão, conexidade fuzzy, auxílio computadorizado ao diagnóstico.

\section{COMPUTER AID TO THE IDENTIFICATION OF LUNG CANCER BASED ON MAPS CONNECTEDNESS FUZZY STARTING FROM THE CHOICE OF A IDEAL SEED}

\begin{abstract}
The objective of this study was to evaluate both qualitatively the accuracy of the segmentation through the algorithm for map construction of fuzzy connectedness based on the choice of an ideal seed compared to manual segmentation. We randomly selected 30 nodules of the project "Lung Image Database Consortium". The developed algorithm was effective in identifying lung nodules reaching 90.4\% accuracy. Moreover, it surpassed the accuracy of the traditional segmentation algorithm for region growing at $9.1 \%$. Therefore, this technique has the potential to be used as a tool for the aid to diagnosis of lung cancer.
\end{abstract}

KEYWORDS: image segmentation, lung cancer, fuzzy connectedness, computer aid to diagnosis. 


\section{AUXÍLIO COMPUTADORIZADO À IDENTIFICAÇÃO DO CÂNCER DE PULMÃO BASEADO EM MAPAS DE CONEXIDADE FUZZY PARTINDO DA ESCOLHA DE UMA SEMENTE IDEAL}

\section{INTRODUÇÃO}

O câncer é um crescimento celular anormal, incontrolável, que invade tecidos vizinhos e os destroem. Somente nos Estados Unidos em 2005, foram registrados mais de 175 mil casos de câncer do pulmão que levaram a morte mais de 160 mil pessoas. Este total é tão expressivo que supera o número de óbitos provocados pela soma dos 3 tipos de câncer de maior incidência (mama, colorretal e próstata). Segundo dados do Instituto Nacional do Câncer - INCA (www.inca.org.br), o número de novos casos do câncer de pulmão estimados para o Brasil no ano de 2008 foi de 17.810 entre homens e de 9.460 para mulheres. Entretanto, pacientes que são diagnosticados precocemente, logo nos primeiros meses da doença, possuem $60 \%$ a $90 \%$ de chance de sobrevivência [Bellotti e Carlo 2007]. Logo, a detecção e o tratamento precoce do câncer de pulmão são formas efetivas de garantir a vida dos pacientes. Todavia, a detecção e a classificação dos nódulos em benigno ou maligno são tarefas desafiadoras aos especialistas, pois os nódulos são pequenos, apresentam baixo contraste e normalmente estão inclusos em estruturas anatômicas complexas. A segmentação do nódulo pulmonar é tipicamente realizada de forma manual, por um médico especialista. Porém, a extração manual do contorno do nódulo é tediosa, consome muito tempo e sofre de variações intra e inter-observador, necessitando assim de uma forma rápida e precisa para identificar nódulos pulmonares. Logo a implementação de um método semi-automático de segmentação favorece o diagnóstico precoce do câncer de pulmão aumentando a chance de sobrevivência do paciente.

Diante deste contexto, diversos métodos de auxílio computadorizado ao diagnóstico (CAD) foram propostos na literatura para assistir os especialistas em detectar o câncer de pulmão precocemente. A segmentação é uma das principais técnicas de processamento de imagem utilizadas no CAD, pois permite ao especialista separar o(s) nódulo(s) suspeito(s) da complexa estrutura anatômica pulmonar [Yoo 2004]. Além disso, permite analisar o volume do nódulo de maneira quantitativa e qualitativa por meio da reconstrução $3 \mathrm{D}$ que permite mensurar a gravidade e a evolução do nódulo mais facilmente. Uma técnica bastante utilizada na segmentação de imagens do pulmão é a de Crescimento de Região [Sluimer e Schilham 2006]. A literatura apresenta índices de precisão na segmentação de nódulos pulmonares isolados de até 68,5\% [Sonka, Park e Hoffman 2008]. Todavia, um método ouro para a segmentação precisa dos diversos tipos de nódulos permanece um problema cujo qual a literatura não resolve. Dehmeshki et al. [Dehmeshki e Amin 2008] propuseram um algoritmo baseado no crescimento de região limitado por um mapa de conexidade fuzzy utlizando o critério de busca por uma semente ideal. O método foi capaz de segmentar diversos tipos de nódulos com precisão média de $80 \%$. Os tipos de nódulos segmentados foram: os anexos a estruturas vasculares, nódulos próximos ao diafragma e também os que apresentavam baixo e alto contraste. Contudo, os resultados foram obtidos adotando uma avaliação qualitativa dos resultados pelos especialistas, que em muitos casos pode ser tendenciosa.

Logo, o objetivo principal deste trabalho foi comparar de maneira quantitativa a precisão da segmentação por meio do algoritmo de construção de mapas de conexidade fuzzy partindo da escolha de uma semente ideal em relação à segmentação manual. Como 
objetivo secundário pode-se citar o teste da hipótese de que os algoritmos de crescimento de região produzem resultados mais precisos quando direcionados pela conexidade fuzzy na segmentação de nódulos pulmonares.

\section{MATERIAIS E MÉTODOS}

Os algoritmos computacionais foram desenvolvidos utilizando a linguagem de programação Java 1.6 no sistema operacional Linux Ubuntu 10.04. Este trabalho utilizou as imagens de uso público do projeto "Lung Image Database Consortium" (LIDC). O LIDC contém 90 exames com 829 nódulos malignos e benignos. Cada exame possui em média 200 imagens DICOM ("Digital Imaging Communications in Medicine") de Tomografia Computadorizada (CT) de Tórax. Um arquivo XML descreve as posições cartesianas da segmentação manual realizada por um especialista dos nódulos e a classificação de cada nódulo pertencente ao exame [Mcnitt-Gray e Armato 2007].

Neste trabalho foram desenvolvidas as técnicas de segmentação utilizando apenas o algoritmo de crescimento de região e a segmentação por crescimento de região orientada por mapas de conexidade fuzzy. Foram selecionados de maneira aleatória 30 nódulos do LIDC. Para cada nódulo foi selecionado o seu corte mediano para aplicar os algoritmos. Os testes dos algoritmos foram aplicados dez vezes para cada nódulo buscando demonstrar consistência entre os resultados, pois como os algoritmos são semi-automáticos estes necessitam de ajustes no nível de segmentação para poder assim alcançar uma precisão satisfatória. Assim, neste trabalho foram avaliadas as precisões dos métodos de segmentação semi-automática tendo como base a segmentação manual. A precisão é obtida por meio do cálculo da área em pixels resultante da subtração de imagens entre os nódulos segmentados, via segmentação automática ou manual.

O trabalho foi baseado em três etapas de desenvolvimento: interface, leitura da imagem DICOM e a principal, que é a segmentação da imagem utilizando os algoritmos de Crescimento de Região e Conexidade Fuzzy. A interface oferece algumas opções, dentre elas estão o leitor e visualizador de imagens no padrão DICOM e as ferramentas básicas de processamento de imagens (zoom, translação e janelamento).

A partir da interface gráfica (Figura 1 - A) o especialista seleciona o arquivo de imagem DICOM de interesse, ao ser exibida a imagem o especialista seleciona a região de interesse e "planta" uma semente (pixel base) de onde o algoritmo de busca por uma semente ideal tem início, a seleção dessa região de interesse (ROI) é feita por meio do mouse. Também na interface existe um slider (Figura 1 - B) que controla o nível de segmentação da imagem de acordo com os critérios da Afinidade Fuzzy. Para facilitar a interação do especialista, os valores de afinidade foram normalizados entre 0 e 1 . Desta forma, quanto mais próximo do valor 0 maior o número de ramificações no nódulo, caso contrário, quando se aproxima mais do valor 1 o número de ramificações diminui ocasionando uma segmentação mais contida.

Desta forma o especialista encontra mais recursos não só por meio dos algoritmos de crescimento de região e conexidade Fuzzy, mas também por meio do slider para um maior controle no nível de segmentação, conseguindo assim uma maior precisão e detalhamento sobre o nódulo a ser segmentado. 


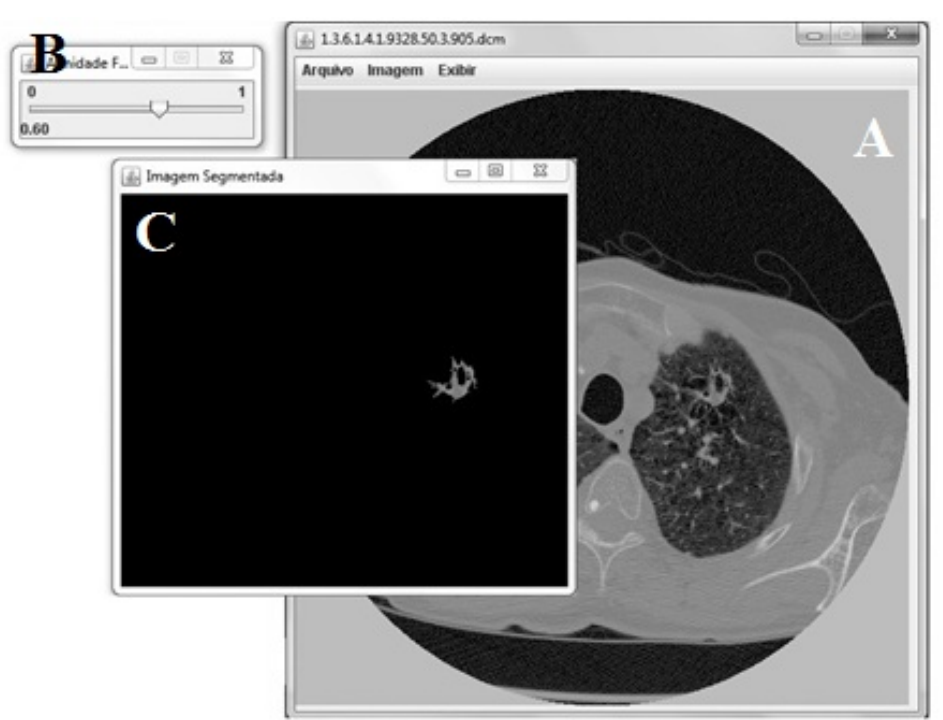

Figura 1 - (A) Interface de execução do algoritmo de segmentação; (B) Slider de ajuste; (C) Resultado da segmentação.

\section{BUSCA POR UMA SEMENTE IDEAL}

Devido à complexidade da anatomia e a dificuldade do especialista em definir a melhor "semente", foi desenvolvido um algoritmo de busca capaz de identificar uma "semente" ideal de acordo com a semente inicial que foi selecionada pelo usuário, esta semente ideal é um pixel que possui um nível de intensidade maior do que a dos seus vizinhos, este método foi implementado em forma de uma busca em profundidade em um grafo, efetuando a comparação dos quatro vizinhos para assim poder verificar se estes têm relação de conectividade. Por exemplo, um pixel $p$ nas coordenadas $(x, y)$ possui dois vizinhos horizontais e dois verticais, cujas coordenadas são dadas por $(x+1, y),(x-1, y),(x$, $y+1),(x, y-1)$.

Segundo [Gonzalez e Woods 2002], a conectividade entre pixels é um conceito importante usado no estabelecimento das bordas de objetos e componentes de regiões em uma imagem. Para estabelecer se dois pixels estão conectados, é preciso determinar se eles são de alguma forma adjacentes (digamos, se são vizinhos-de-4) e se seus níveis de cinza satisfazem um certo critério de similaridade (digamos, se eles são iguais). Por exemplo, em uma imagem binária com valores 0 e 1 , dois pixels podem ser vizinhos-de- 4 , mas eles não são ditos conectados a menos que tenham o mesmo valor.

\section{CONEXIDADE FUZZY}

As funções conhecidas como fuzzy permitem quantificar o quão próximo dois pixels estão e quanto parecidos eles são em relação a propriedades baseadas nos brilhos de pixels presentes em vizinhanças dos pixels considerados. $\mathrm{O}$ valor de conexidade entre dois pixels $p$ e $q$ é dado pela maior força de conexidade entre todos os caminhos possíveis de $p$ a $q$ na imagem. A força de conexidade de um caminho é definida como sendo o valor do elo mais fraco neste caminho, ou seja, a menor afinidade entre pixels vizinhos que estejam neste caminho. A conexidade fuzzy permite a segmentação de objetos irregulares e complexos, pois é fortemente baseado na intensidade do pixel, assim, pode utilizar o valor da conexidade para direcionar de forma mais acurada o algoritmo de crescimento de região [Zhou e Bai 2007]. 
A segmentação fuzzy é uma técnica que determina para cada elemento da imagem um grau de pertinência (entre zero e um) indicando a certeza dele pertencer (ou não) a um determinado objeto (que acredita-se estar contido na imagem) [Herman e Carvalho 2001]. Essas informações de pertinência possibilitam que o algoritmo segmente a imagem tomando uma decisão mais flexível acerca da classificação de cada elemento. A semente ideal dará início ao algoritmo de crescimento de região, que também usa a vizinhança-de-4 para verificar e atribuir os pesos aos pixels da imagem construindo assim um mapa de conexidade entre os pixels da imagem com o auxílio de uma função, esta função pode ser entendida como uma função de afinidade fuzzy que irá determinar o quanto um pixel pertence à região de interesse numa escala de 0 a 1 , onde o valor 1 corresponde a pertinência total (pixel semente ideal da segmentação) e o valor 0 como não pertencendo a região. Um pixel $p$ é dito conectado ao pixel semente se este pixel $p$ obtiver um nível de afinidade forte, ou seja, se o seu grau de pertinência (de 0 a 1) satisfizer as condições para pertencer a uma determinada região de interesse, este processo é conhecido como Conexidade Fuzzy.

\section{O ALGORITMO}

Sendo o espaço digital denominado pelo par ordenado $(V, \pi)$, tal que $V$ é um conjunto conectado por $\pi$. Os elementos de $V$ são referidos por spels ("spacial elements"), spels podem representar pontos em um plano, pixels em uma imagem ou voxels em um volume. Uma afinidade fuzzy do spel é uma função $\psi: \mathrm{V}^{2} \rightarrow[0,1]$, esta atribui valor entre 0 e 1 para cada par ordenado de spels, tal que:

1) Para todo $\in, \psi(c, c)=1$, e

2) Para todo, $\in, \psi(\mathrm{c}, \mathrm{d})=\psi(\mathrm{d}, \mathrm{c})$.

Para fazer a análise de cada spel e encontrar o grau de pertinência que esse spel tem com cada objeto da imagem é utilizado o conceito de conexidade fuzzy [Herman e Carvalho 2001], conforme definida a seguir:

1) Uma corrente de de $c^{(0)}$ a $c^{(\mathrm{k})}$ corresponde a uma sequência arbitrária de $\left(c^{(0)}, \ldots\right.$, $\left.c^{(\mathrm{k})}\right)$ de spels distintos;

2) O par ordenado $\left(c^{(\mathrm{k}-1)}, c^{(\mathrm{k})}\right)$ de spels consecutivos corresponde a um elo na corrente;

3) $\psi(c(k-1), c(k))$ representa a força do elo.

Consequentemente, a conexidade fuzzy de um par de spels $(c, d)$ é a força $\psi$ da corrente mais forte de $c$ a $d$. No caso de uma determinada corrente possuir somente um spel, a sua força $\psi$ é definida como sendo 1 [Cosme 2008]. As funções $\psi$ calculam as afinidades entre spels e, após o processo de segmentação, fornecem o grau de confiança que dois spels possuem em pertencer a uma mesma classe. Equação 1 faz a atribuição de um grau de pertinência ou afinidade fuzzy $\psi$ que um spel $\beta$ tem com a semente ideal $\alpha$, a partir desta função de afinidade é obtido o mapa de conexidade fuzzy da região de interesse.

$$
\psi=\beta / \alpha
$$

\section{LIMITAR O MAPA DE CONEXIDADE}

No mapa de conexidade pode ser definido um limiar para assim poder obter como resultado a segmentação da imagem. 
O valor adotado como padrão é em 0.5 , mas podendo ser modificado pelo especialista dentro da escala normalizada de 0 a 1 por meio do slider (Figura $1-$ B). Lembrando que quanto mais próximo do valor 0 maior o número de ramificações no nódulo, caso contrário, quando se aproxima mais do valor 1 o número de ramificações diminui ocasionando uma segmentação mais contida.

A partir disto é possível obter uma segmentação mais precisa do nódulo. Importante salientar que a definição deste limiar só é feita após a construção do mapa de conexidade Fuzzy.

\section{PSEUDOCÓDIGO}

Abaixo segue a descrição geral do algoritmo da conexidade fuzzy segundo [Sonka, Hlavac e Boyle 2008]:

1. Definir propriedades de adjacência e afinidade fuzzy;

2. Determinar os valores de afinidade para todos os pares de spels ("spacial elements" ou pixels) adjacentes;

3. Determinar a semente (pixel semente ideal) da segmentação;

4. Determinar todos os caminhos possíveis entre a semente e todos os outros elementos da imagem, dentro do domínio da imagem (sem formação de loops) considerando o relacionamento da adjacência fuzzy;

5. Para cada caminho, determinar a força deste caminho de acordo com a afinidade mínima dos próximos caminhos;

6. Para cada elemento da imagem, determinar a conexidade fuzzy entre este elemento e a semente conforme a força máxima de todos os possíveis caminhos e como resultado formar o mapa de conexidade desta imagem;

7. Limitar o mapa de conexidade com um limiar apropriado para segmentar a imagem dentro de um objeto contendo a semente e o "background".

\section{RESULTADOS E DISCUSÃO}

A segmentação fuzzy de imagens tem se mostrado eficaz nos casos em que a característica que distingue o objeto de interesse do "background" é alguma propriedade textural, ao invés do exato valor atribuído aos pixels, como por exemplo, imagens contendo sombras, iluminação não uniforme e/ou ruídos [Herman e Carvalho 2001]. A presença dessas interferências em uma imagem requer dos algoritmos de segmentação um esforço adicional durante o reconhecimento dos objetos presentes na mesma. Devido a estas características a conexidade fuzzy tem-se tornado uma promissora técnica de auxílio ao diagnóstico do câncer de pulmão, visto que, soluciona as principais problemáticas intrínsecas do nódulo como o baixo contraste e a inclusão junto a estruturas.

Neste trabalho foi desenvolvida uma ferramenta de código livre para auxiliar especialistas na identificação de nódulos pulmonares. $\mathrm{O}$ banco de nódulos desenvolvido obedece à estrutura do arquivo XML criado pelo LIDC. Dessa forma, exames futuros inseridos no banco obedecerão às especificações do projeto LIDC. Isto permitirá a padronização da base de nódulos e, logo, a colaboração com os idealizadores do projeto.

O sistema desenvolvido permitiu ao especialista visualizar em detalhes o resultado da segmentação obtida por meio de uma interface gráfica (Figura 1 - C). Assim, caso a 
segmentação não tenha sido satisfatória ele pode retornar para a interface de segmentação e definir novos parâmetros à segmentação.

A segmentação semi-automática utilizando a Conexidade Fuzzy partindo da escolha de uma semente ideal obteve precisão média de $90,4 \%$ e superou em $9,1 \%$ a precisão do algoritmo de Crescimento de Região (Tabela 1). Na figura 2 é ilustrado o resultado da segmentação de três nódulos malignos utilizando a segmentação manual e os algoritmos semi-automáticos.

Tabela 1: Médias dos níveis de precisão dos algoritmos de segmentação.

\begin{tabular}{|l|r|r|r|}
\hline & \multicolumn{1}{|c|}{$1^{\circ}$ Grupo } & $2^{\circ}$ Grupo & $3^{\circ}$ Grupo \\
\hline Crescimento de Região & $86,6 \%$ & $79,9 \%$ & $78,4 \%$ \\
\hline Conexidade Fuzzy & $98,4 \%$ & $84,1 \%$ & $88,8 \%$ \\
\hline
\end{tabular}

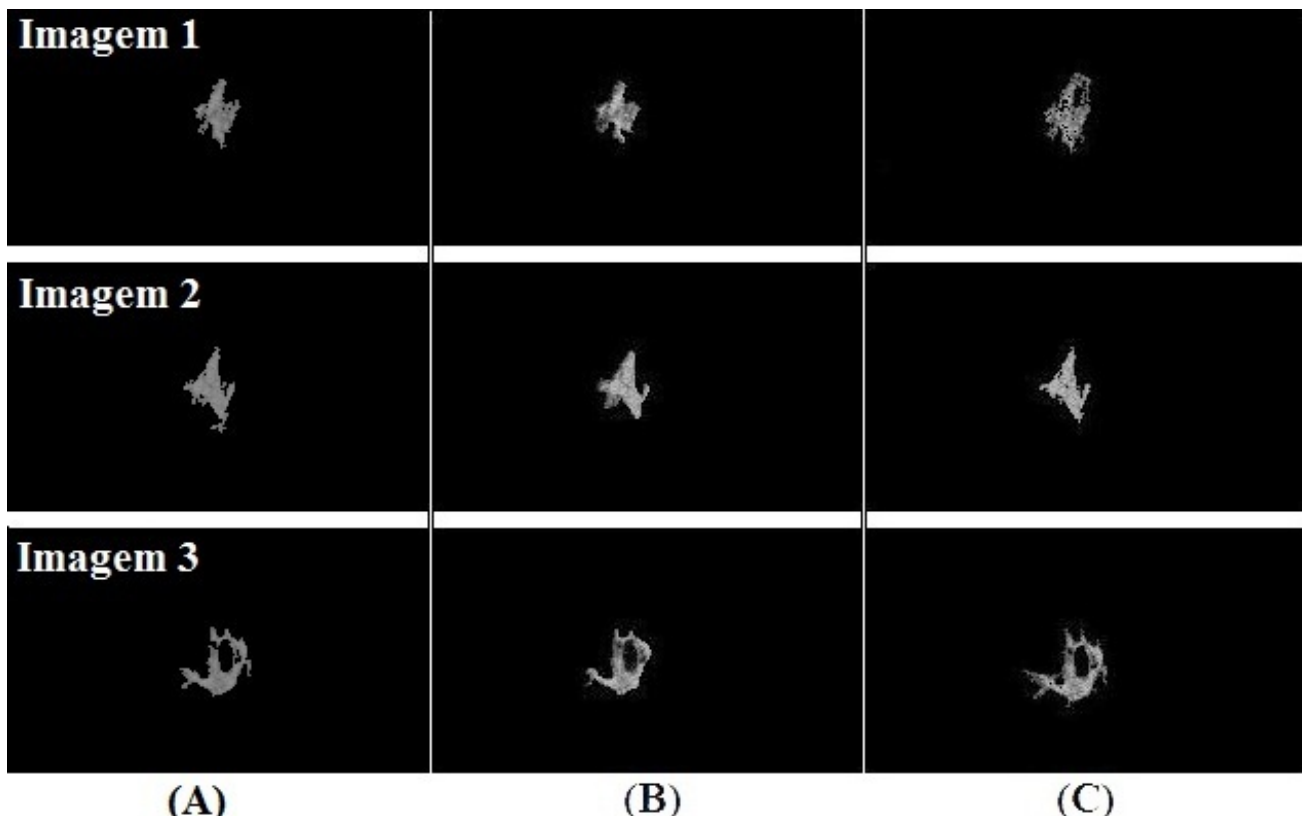

(A)

(B)

(C)

Figura 2 - Imagens segmentadas pelos diferentes métodos. Em colunas: (A) Conexidade Fuzzy; (B) Manual; (C) Crescimento de Região.

A Tabela 1 apresenta diferentes níveis de precisão para cada um dos grupos de imagens, isto ocorre em virtude da variedade dos tipos de nódulos segmentados, nódulos que se encontram junto à parede pulmonar, anexos a estruturas vasculares ou ao diafragma. A tarefa de segmentar o nódulo que se encontra junto à parede pulmonar é bem desafiadora, pois se torna consideravelmente difícil identificar a região do nódulo da região pertencente à parede pulmonar, isto é devido à semelhança quase que indistinguível dessas duas regiões, a segmentação neste caso é de complexidade considerada de nível alto. Já no caso em que o nódulo encontra-se junto a estruturas vasculares, a segmentação é considerada em nível de complexidade mediano, pois as características que distinguem o nódulo das estruturas vasculares (intensidade do pixel e conexidade) tornam-se mais evidentes. Portanto, como estes dois casos diferenciam em termos de complexidade da segmentação, os níveis de precisão entre os métodos de segmentação tendem também a ser diferentes, como já foi mencionada e explicada a conexidade fuzzy não faz uso somente do algoritmo de crescimento de região, mas também da criação de um mapa de conexidade fuzzy partindo da escolha de uma semente ideal o que a faz obter níveis de precisão superiores a 
segmentação que utiliza apenas o algoritmo de crescimento de região. Esta variedade nos tipos de nódulo foi de importância fundamental para a análise dos diferentes métodos de segmentação.

\section{CONCLUSÃO}

O algoritmo de construção de mapas de conexidade fuzzy partindo da escolha de uma semente ideal mostrou níveis bastante satisfatórios, isto para diferentes tipos de imagens. $\mathrm{O}$ algoritmo obteve resultados próximos ao da segmentação realizada de forma manual pelo especialista. Além disso, em todos os casos avaliados o algoritmo obteve precisão superior a técnica de segmentação que utiliza apenas o algoritmo de crescimento de região. A conexidade fuzzy foi capaz de isolar o nódulo suspeito, excluindo demais estruturas anatômicas que não pertenciam ao mesmo. A ferramenta desenvolvida neste trabalho possui potencial para ser utilizada como uma ferramenta de auxílio ao diagnóstico do câncer de pulmão. É de código livre, fácil uso e multiplataforma.

Iremos estender as funcionalidades do algoritmo para atuar na segmentação tridimensional dos nódulos pulmonares.

\section{REFERÊNCIAS BIBLIOGRÁFICAS}

1. AYRES, P. A. A., CAVAlCANTE, R. C., OliveirA, M. C. (2010) "Auxílio à Classificação de Nódulos Pulmonares Usando Recuperação de Imagens Similares Baseada em Análise de Textura 3D e Registro de Imagem 3D”, XXX Congresso da Sociedade Brasileira de Computação - WIM - X Workshop de Informática Médica, Belo Horizonte/MG - Brasil.

2. COSME, I. C. S., "Utilizando Mapas de Conectividade Fuzzy no Desenvolvimento de Algoritmos Reparadores de Imagens Binárias 3D", Centro de Ciências Exatas e da Terra - UFRN, Natal-RN, 2008.

3. G. T. HERMAN AND B. M. CARVALHO. (2001) "Multiseeded segmentation using fuzzy connectedness", IEEE Transactions on Pattern Analysis and Machine Intelligence, p. 460-474.

4. GONZALES AND R. WOODS. (2002) "Digital Image Processing", Upper Saddle River - NJ: Prentice-Hall, $2^{\text {th }}$ edition.

5. INCA - Instituto Nacional do Câncer. (2010) “www.inca.org.br".

6. J. DEHMESHI, H. AMIN, M. VALDIVIESO, AND X. J. YE. (2008) "Segmentation of pulmonary nodules in thoracic CT scans: A region growing approach", IEEE Transactions on Medical Imaging, vol. 27, p. 467-480.

7. M. F. MCNITT-GRAY, S. G. ARMATO, C. R. MEYER, A. P. REEVES, G. MCLENNAN, R. C. PAIS, J. FREYMANN, M. S. BROWN, R. M. ENGELMANN, P. H. BLAND, G. E. LADERACH, C. PIKER, J. GUO, Z. TOWFIC, D. P. Y. QING, D. F. YANKELEVITZ, D. R. ABERLE, E. J. R. VAN BEEK, H. MACMAHON, E. A. KAZEROONI, B. Y. CROFT, AND L. P. CLARKE. (2008) "The Lung Image Database Consortium (LIDC) data collection process for nodule detection and annotation," Academic Radiology, vol. 14, pp. 1464-1474. 
8. M. SONKA, W. PARK, AND E. A. HOFFMAN. (1996) "Rule-based detection of intrathoracic airway trees," IEEE Trans. Med. Imag, vol. 15, no. 3, p. 314-326.

9. MILAN SONKA, VACLAV HLAVAC, AND ROGER BOYLE. (2008) "Image Processing, Analysis, and Machine Vision", Thomson, $3^{\text {th }}$ edition.

10. R. BELlOTti, F. DE CARLO, G. GARGANO, S. TANGARO, D. CASCIO, E. CATANZARITI, P. CERELlO, S. C. CHERAN, P. DELOGU, I. DE MITRI, C. FULCHERI, D. GROSSO, A. RETICO, S. SQUARCIA, E. TOMMASI AND B. GOLOSIO. (2007) "A CAD system for nodule detection in low-dose lung CTs based on region growing and a new active contour model," Medical Physics, vol. 34, p. 49014910.

11. SLUIMER, I., A. SCHILHAM. (2006) "Computer analysis of computed tomography scans of the lung: A survey." IEEE Transactions on Medical Imaging, p. 385-405.

12. T. YOO. (2004) "Insight into images: principles and practice for segmentation, registration and image analysis", vol. 1, AK Peters LTDA, Massachusetts, USA.

13. Y. X. ZHOU AND J. BAI. (2007) "Atlas-based fuzzy connectedness segmentation and intensity nonuniformity correction applied to brain MRI", IEEE Transactions on Biomedical Engineering, vol. 54, p. 122-129. 